\title{
Norois
}

Environnement, aménagement, société

\section{Les populations périurbaines face à l'automobile en grande couronne francilienne}

Population and automobile dependance in the outer sububs of Paris

\section{Benjamin Motte-Baumvol}

\section{(2) OpenEdition}

\section{Journals}

Édition électronique

URL : http://journals.openedition.org/norois/1251

DOI : 10.4000/norois. 1251

ISBN : 978-2-7535-1553-6

ISSN : $1760-8546$

Éditeur

Presses universitaires de Rennes

Édition imprimée

Date de publication : 1 décembre 2007

Pagination : 53-66

ISBN : 978-2-7535-0616-9

ISSN : 0029-182X

\section{Référence électronique}

Benjamin Motte-Baumvol, « Les populations périurbaines face à l'automobile en grande couronne francilienne », Norois [En ligne], 205 | 2007/4, mis en ligne le 01 décembre 2009, consulté le 02 mai 2019. URL : http://journals.openedition.org/norois/1251 ; DOI : 10.4000/norois. 1251 


\title{
LES POPULATIONS PÉRIURBAINES FACE À L’AUTOMOBILE EN GRANDE COURONNE FRANCILIENNE
}

\author{
Benjamin Motte-Baumvol \\ Laboratoire THÉMA \\ (Université de Bourgogne), \\ 2 boulevard Gabriel - 21000 Dijon \\ benjamin.motte@u-bourgogne.fr
}

\section{RÉSUMÉ}

Ce travail se propose d'identifier si l'automobile qui a participé au développement des espaces périurbains et qui est porteuse de fortes disparités de mobilité entre les ménages, notamment en fonction de leurs revenus et de leur âge, pourrait conduire à l'exclusion d'une partie croissante de la population. On pense notamment aux populations nonmotorisées mais aussi aux ménages motorisés, les plus modestes ou les plus âgés, qui font difficilement face aux coûts ou aux exigences de la mobilité automobile. Nos résultats montrent que le nombre des ménages non-motorisés, les plus fortement marginalisés, diminue notamment dans les territoires périurbains les plus dépendants de l'automobile, malgré le maintien d'un niveau de chômage élevé et un vieillissement accru des populations. Ce constat qui va à l'encontre du développement d'un périurbain de "relégation » est toutefois nuancé par la mise en évidence, dans certains territoires, des fortes disparités de mobilité que subissent les ménages modestes, pourtant motorisés, par rapport aux ménages les plus favorisés.

MotS CLÉ : Dépendance automobile - Grande couronne - Ille-de-France-Mobilité - Motorisation - Périurbain-Relégation.

\section{ABSTRACT}

\section{Population and automobile dependance in the outer sububs of Paris}

The present article is attempting to identify if the automobile, which took part in development of periurban spaces but which causes strong disparities of mobility between households, according to their incomes and of their age, leads to exclude an increasing part of the population in this spaces. We looked more closely into not-motorized population but also motorized households, of modest income or older age, for which cost or physical requirements of automobile mobility are too high. Our results show that number of notmotorized households, most strongly marginalized, decrease in particular in periurban territories most dependent on car, in spite high level of unemployment and ageing of population. This statement goes against idea of their relegation in periurban territories; however it is moderated, in any territories, by strong disparities of mobility between modest income and high income households.

KEY WORDS : Automobile Dependence-Automobile Equipment-Île-de-France-Mobility - Outer Suburbs - Relegation. 
La place croissante et dominante de la voiture de même que l'évolution des modes de vie tendent à peser chaque jour un peu plus sur les localisations des hommes et des activités, sous l'influence d'une mobilité aisée : l'« automobilité » (Kaufmann et Guidez, 1998). On a observé avec le développement de l'automobile une déconnexion croissante entre lieux de résidence, lieux d'emploi et localisation des diverses ressources nécessaires à la vie quotidienne. M. Berger (2004) note l'augmentation de plus d'un tiers des distances des navettes domicile-travail des franciliens entre 1975 et 1999. De même, à partir de l'inventaire communal, l'INSEE (2001) observe le recul, ces vingt dernières années, de nombreux services et principalement des services de proximité dans une tendance continue depuis 40 ans. J.-P. Orfeuil (2004) estime ainsi, concernant les services, que « la distance-temps en voiture a tendance à se substituer à la distance-temps à pied, entraînant des évolutions fortes dans le maillage et la localisation des commerces et services ».

Cette déconnexion entre localisations et résidences, des activités et des services a été particulièrement forte dans les territoires où la croissance urbaine a été concomitante à la diffusion progressive de l'automobile. Dans les espaces périurbains en particulier, les navettes domicile-travail sont plus longues (Berger, 2004), les services de proximité plus rares (INSEE, 2001) et les alternatives modales moins nombreuses. La voiture y dispose d'un monopole radical au sens où l'entend I. Illich (1973); elle disqualifie les autres modes en les surpassant notamment par ses vitesses de déplacement. Pour G. Dupuy (1999), le monopole radical imposé par l'automobile se manifeste par l'exclusion d'une frange entière de la population notamment les plus pauvres et les plus âgés. Ces populations ne seraient plus ainsi en mesure d'exercer leurs activités (travail, courses, visites à la famille, etc.), ni satisfaire leurs aspirations, si elles ne disposent pas d'une voiture particulière, ou plus largement, si elles ne peuvent s'en servir au moment où le besoin s'en fait sentir.

S’il paraît indéniable que la possession d'une voiture introduit une différence significative entre les ménages motorisés et non-motorisés, en termes de mobilité et d'accessibilité, elle ne constitue pas pour autant une garantie de tirer profit de l'ensemble des possibilités offertes par l'« automobilité ». L. Hivert et J. Péan de Pontfilly (2000) ont montré que la faiblesse de leurs revenus pouvait conduire les ménages motorisés à limiter le niveau de leur mobilité automobile. Certains ménages, notamment les plus modestes, feraient ainsi difficilement face aux fortes « injonctions à la mobilité automobile » (Wenglenski, 2003) inhérentes aux territoires périurbains.

La question qui se pose dans ce travail est donc d'identifier si l'automobile qui a participé au développement des espaces périurbains et qui est porteuse de fortes disparités de mobilité entre les ménages, notamment en fonction de leurs revenus et de leur âge, pourrait conduire à l'exclusion d'une partie croissante de la population, dans la mesure où l'on considère la mobilité comme une condition de la socialisation (Godard, 2001). Cette hypothèse prend un relief particulier dans un contexte de développement d'un périurbain de « relégation » (Guilluy et Noyé, 2004; Rougé, 2005), c'est-à-dire l'émergence de territoires périurbains dans lesquels tendent à être rejetées des populations modestes ou vieillissantes ne pouvant accéder au sol urbain dans les centres, mais également de maintien d'un niveau de chômage élevé et d'un vieillissement prononcé de la population.

Pour répondre à ce questionnement, nous nous arrêterons tout d'abord sur les populations qui font le plus difficilement face à la dépendance à l'automobile dans les espaces périurbains : les populations non-motorisées. Nous verrons notamment s'ils tendent à constituer une population croissante en situation de forte exclusion. Puis, nous examinerons pour les ménages motorisés l'ampleur des disparités auxquelles ils sont confrontés selon leur niveau de revenu et leur âge. Enfin, nous simulerons trois scénarios pour éclairer les futurs possibles et évaluer les perspectives d'action à même d'alléger la pression automobile qui pèse sur les populations périurbaines. Mais avant cela nous présenterons dans une première partie notre terrain d'étude

\section{Les périurbains d'île-de-France}

Le territoire d'étude de cet article porte spécifiquement sur la région Île-de-France pour laquelle nous disposions de données qui sont à la base de cette recherche : il s'agit des données de l'En- 
quête Globale de Transport (EGT) ${ }^{1}$. Pour autant, notre étude n'a pas porté sur l'ensemble de ce territoire administratif, nous nous sommes limités à ses quatre départements périphériques, soit la grande couronne francilienne où une partie substantielle des espaces périurbains franciliens.

En effet, la définition du périurbain que nous avons retenu dans ce travail est celle d'un « ensemble de zones où l'on observe des phénomènes de croissance démographique... où l'on enregistre une production de logements neufs sous des formes variées » (Jalabert, 1985). Selon cette définition, qui n'est pas si loin de celle de M.-C. Jaillet (1985) pour qui le périurbain est « une fraction de l'espace sur laquelle se localise... une nouvelle phase de la croissance urbaine, plus adaptée à l'état du système socio-économique... », le périurbain francilien déborde donc de l'Île-de-France, mais couvre dans la région la majeure partie des territoires de grande couronne. Ce sont ces espaces où les dynamiques des populations et des activités sont particulièrement fortes (Massot et Roy, 2004). Ils absorbent la quasi-totalité de la croissance démographique ${ }^{2}$ et la majeure partie de celle du parc de logements entre 1990 et 1999 (Carre, 2005), tandis que dans les autres départements la population reste relativement stable, la construction de logements est moins vive et la croissance urbaine y est plus ancienne.

Notre acceptation relativement large des espaces périurbains, nous invite à ne pas les considérer comme des espaces homogènes. Ainsi, en termes de mobilité et de dépendance à l'automobile les contextes territoriaux en grande couronne sont fortement contrastés, comme l'ont montré S. Wenglenski (2003) pour l'accès à l'emploi et B. Motte (2006) pour l'accès aux services aux ménages. C'est pourquoi nous avons travaillé à partir d'une typologie des territoires périurbains classant les communes à partir de quatre niveaux d'offre de services (fig. 1). Cette classification est le résultat d'un travail préalable qui a montré que la présence de services dans un territoire tendait, en fonction de leurs natures, à moduler l'intensité de la dépendance subie par les ménages en jouant sur leur niveau de motorisation (Motte, 2006).

Le premier niveau correspond ainsi aux communes qui ne disposent pas d'une base minimum de services de proximité. Dans le second niveau d'équipement, la présence d'une base minimum de service de proximité est indiquée par la présence d'un magasin d'alimentation générale (MAG). Le troisième niveau est matérialisé par la présence d'une grande surface à dominante alimentaire (GSA) qui a un effet puissant d'agrégation de commerces et services dans ses alentours. Enfin le bouquet de service comprend à la fois tous les équipements retenus précédemment auxquels s'ajoutent des services de santé (pharmacie, médecin généraliste), un bureau de poste, dans la plupart des cas une banque, des magasins spécialisés et au moins une grande surface spécialisée. Ces services sont ceux que fréquente régulièrement un ménage, c'est-à-dire au moins une fois par mois.

Le résultat de notre typologie montre, en grande couronne francilienne, une forte concentration des services là où les densités de population sont les plus fortes. On constate également que si plus de la moitié des communes de notre territoire d'étude ne disposent pas d'une base minimum de services de proximité, elles ne regroupent que moins de $10 \%$ de la population de la zone.

1. L’EGT est une enquête sur la mobilité des ménages franciliens. Elle est menée depuis 25 ans (1976, 1983, 1991, et 2001) sur un échantillon de près de 10500 ménages, sous la direction de la Direction Régionale de l'Équipement d'Île-de-France. Le principe de recueil est celui des enquêtes de déplacements menées classiquement dans les grandes agglomérations françaises. Les ménages sont enquêtés en face à face. Chacun des membres du ménage de six ans ou plus est interrogé sur tous les déplacements qu'il a effectués la veille du jour de l'enquête. Pour chacun de ses déplacements un nombre important de caractéristiques est recueilli : motif du déplacement, mode(s) de transport utilisé(s), origine, destination, distance, durée etc.

2. Source : site internet de l'INSEE dans la rubrique des chiffres clés. 


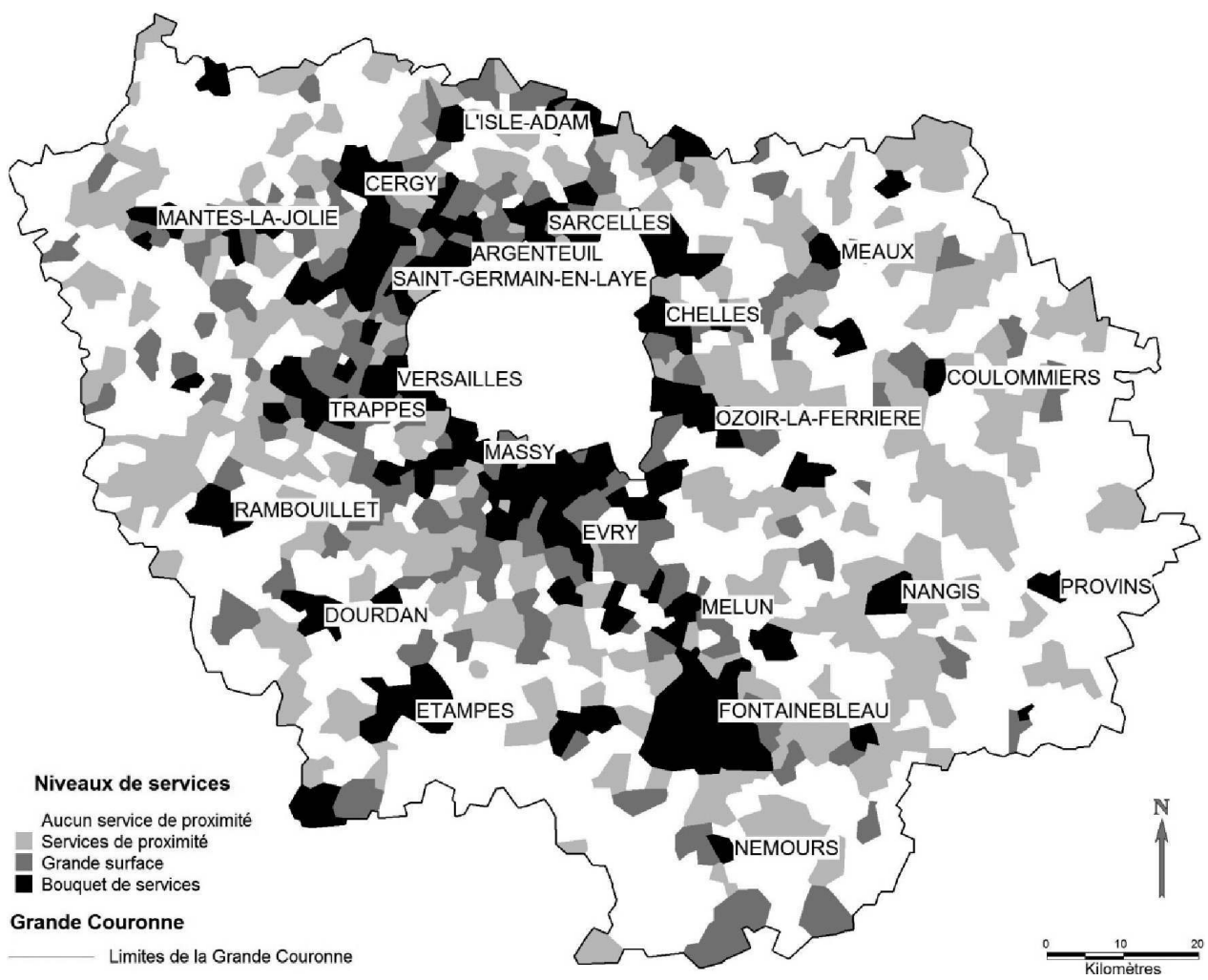

Figure 1 : Typologie des communes de grande couronne francilienne (Source : Inventaire communal 1998; réalisation de l'auteur)

Typology of local services in outer suburbs from Paris

\section{Les ménages les plus menacés face à la dépendance automobile reculent notamment dans les territoires les plus exposés}

\section{DES MÉNAGES NON-MOTORISÉS FORTEMENT MARGINALISÉS DANS LES ESPACES PÉRIURBAINS}

Les populations non-motorisées ont une mobilité fortement marquée par leur absence d'équipement automobile. C'est tout d'abord en termes de nombre de déplacements par jour que s'expriment les différences par rapport aux ménages motorisés. Les adultes des ménages non-motorisés font en moyenne un déplacement de moins par jour par rapport aux adultes appartenant aux ménages motorisés et cet écart est le plus important dans les communes pas ou peu équipées en services. De plus parmi les ménages non-motorisés on relève que $15 \%$ des personnes n'effectuent aucun déplacement un jour de semaine contre seulement $6 \%$ dans les ménages motorisés. Enfin, les différences de mobilité entre ces ménages s'expriment en termes de portée des déplacements qui varient du simple au double. Pour autant, le nombre de déplacements plus réduit sur des distances beaucoup plus courtes des adultes non-motorisés s'effectue à budget temps sensiblement équivalent à celui des adultes motorisés. Ceci traduit clairement l'impossibilité pour les personnes appartenant à un ménage non-motorisé d'avoir une mobilité similaire à celle des personnes disposant d'une voiture et ce tout particulièrement dans les communes les plus dépendantes de l'automobile. 
Si la mobilité des membres des ménages non-motorisés est fortement réduite et contrainte par leur absence d'équipement automobile cela ne leur ôte pas toute possibilité de déplacement même dans les territoires de la grande couronne francilienne pourtant fortement dépendants de l'automobile. Si une part importante des adultes non-motorisés ne se déplace pas un jour de semaine, c'est notamment dû à leur profil particulier. Ce sont essentiellement des retraités (60\%) et/ou des individus souffrant d'une incapacité physique permanente (31\%) ou temporaire (15\%) ne leur permettant pas de se déplacer. Seulement $7 \%$ des individus ne s'étant pas déplacés le jour de l'enquête et appartenant à un ménage non-motorisé n'apportent aucune explication à cette absence de mobilité qui peut alors être mise sur le compte de leur absence d'équipement automobile. Cette valeur est significativement plus importante que pour les ménages motorisés (3\%) mais reste modeste.

Pour ceux qui ont effectué un déplacement toute l'étendue des motifs est représentée, notamment les visites aux proches, les loisirs, ou les achats même si ceux-ci sont moins fréquents et ont une portée inférieure. Pour les ménages d'actifs non-motorisés, les déplacements hors travail et de retour au domicile sont limités. Ce sont souvent des déplacements de faible portée (moins d'1 km), à proximité du domicile ou du travail, qui constituent l'unique déplacement des actifs non-motorisés surtout dans les territoires pas ou peu équipées de services. Pour les actifs motorisés, environ deux déplacements de portée moyenne viennent s'ajouter à ceux relatifs au travail. Ces déplacements sont plus nombreux et de plus longues portées. Les différences entre ménages non-motorisés et ménages motorisés se traduisent également par des comportements et des pratiques de mobilité spécifiques fortement différenciées entre les territoires.

\section{L'ABSENCE D'AUTOMOBILE PARTIELLEMENT COMPENSÉE PAR DIVERS ACCOMMODEMENTS}

Les travaux d'Olivier Coutard et al. (2002) sur les conditions de mobilité de ménages pauvres ont révélé, en dehors d'une limitation du nombre et de la portée de leurs déplacements, un ensemble de pratiques permettant aux ménages non-motorisés de faire face aux difficultés liées à la dépendance automobile. Trois types de pratiques ont ainsi été identifiés. La première de ces pratiques concerne le recours à la famille, aux amis ou aux voisins afin de disposer d'une voiture, comme conducteur ou comme passager. Le second type de pratique mis en évidence dans cette étude consiste en un recours plus fréquent aux espaces de proximité. Enfin, le dernier type de pratique correspond à un recours accru aux transports en commun. Cependant l'étude d'O. Coutard et al., par son caractère qualitatif, ne permet pas d'appréhender l'ampleur de ces pratiques.

Selon les analyses que nous avons effectuées en grande couronne francilienne à partir de l'EGT, la majeure partie des ménages non-motorisés aurait accès, de façon au moins hebdomadaire, à une voiture en tant que conducteur ou passager. Ils s'appuient pour cela sur leurs proches qui les conduisent ou mettent à leur disposition leur voiture pour des motifs divers, où dominent le retour au domicile, mais également les motifs affaires professionnelles, achats, affaires personnelles ou loisirs. Ainsi, on peut penser que ces proches appartiennent aussi bien à la famille, aux amis, aux voisins et aux collègues de travail des ménages concernés.

La seconde pratique étudiée est le recours intensif aux espaces de proximité. Ainsi, plus de $60 \%$ des déplacements des ménages non-motorisés se font à l'intérieur de leur commune de résidence et dans un rayon inférieur au kilomètre, contre environ $35 \%$ des déplacements pour les ménages motorisés. Ce recours à l'espace local par les ménages non-motorisés concerne majoritairement l'accès aux commerces et services aux ménages, aussi bien pour les actifs que pour les retraités. Le recours aux territoires de proximité semble donc indispensable à l'approvisionnement des ménages non-motorisés.

Enfin le troisième type de pratique, le recours aux transports en commun, s'applique tout particulièrement aux déplacements liés au travail. Ainsi, plus de 60 \% des actifs non-motorisés se rendent sur leur lieu de travail en transports en commun, contre $20 \%$ des actifs motorisés, y compris 
dans les territoires où l'offre pour ce mode est particulièrement faible ${ }^{3}$. Le recours aux transports en commun paraît incontournable pour les ménages d'actifs non-motorisés alors que les retraités de grande couronne les utilisent très peu (dans $8 \%$ de leurs déplacements).

Ces trois types de pratiques constituent, ensemble, un substitut à l'automobile pour les ménages non-motorisés. On pourrait estimer que ce mode d'organisation des déplacements relève de la « débrouille » et que chaque jour un petit changement menace de remettre en cause l'ensemble de l'édifice. Néanmoins, ce système affiche une certaine robustesse dans la mesure où il permet jour après jour d'assurer une part importante de la mobilité des ménages non-motorisés. Certaines évolutions sont toutefois susceptibles de le menacer ou de le fragiliser. En effet, ces trois formes de dépendances apparaissent complémentaires et donc non substituables l'une à l'autre. De cette manière, si l'un de ces arrangements tombe, la localisation résidentielle des ménages non-motorisés pourrait être remise en question. Le recul des services de proximité, prévisible dans la prochaine décennie, devrait réduire la possibilité de recourir aux ressources locales dans un certain nombre de communes et on voit mal comment le recours aux transports en commun ou à ses proches, peu adapté à des déplacements plus nombreux et surtout plus fréquents, pourraient constituer une solution de substitution. La possibilité de recours à des proches nous parait en effet conditionnée au grand nombre des ménages motorisés, au faible nombre des ménages non-motorisés et à la modestie des sollicitations des seconds envers les premiers.

\section{LE RECUL PARTIEL DES MÉNAGES NON-MOTORISÉS}

Les situations individuelles que nous venons de décrire correspondent à $15 \%$ des ménages de grande couronne. Ce pourcentage varie selon les territoires. On compte moins de $7 \%$ de ménages non-motorisés dans les communes qui ne disposent d'aucun service aux ménages et donc de transports en commun et jusqu’à $20 \%$ des ménages dans les communes les mieux équipées. En 1999, on ne compte que 275000 ménages non-motorisés en grande couronne et parmi eux seuls 8400 résident dans une commune non-équipée de services de proximité. Les ménages non-motorisés sont donc fortement concentrés dans les communes les mieux équipées en services.

La période 1990-1999 montre un recul des ménages non-motorisés en grande couronne. La part de ces ménages est passée de $18 \%$ à $15 \%$. En terme d'effectif cela se traduit par une baisse de 2,5\% du nombre de ménages non-motorisés alors que le nombre total de ménages en grande couronne a augmenté de $12 \%$ au cours de ces dix années. La tendance est donc au recul du nombre et de la part relative des ménages non-motorisés. Toutefois, il faut nuancer ce recul en fonction du statut de ces ménages. La non-motorisation parmi les ménages d'actifs (qui représentent $45 \%$ des ménages non-motorisés pour l'ensemble de la grande couronne) est restée relativement stable, alors qu'elle a fortement diminuée pour les ménages de retraités (qui représentent $40 \%$ des ménages non-motorisés pour l'ensemble de la grande couronne).

Parmi les ménages d'actifs, la part des ménages non-motorisés n'a baissé que d'un point s'établissant à $10 \%$. Plusieurs tendances concernant le statut des actifs et la structure des ménages expliquent la quasi-stagnation du niveau de la non-motorisation pour les actifs. Tout d'abord, on note entre 1990 et 1999 en grande couronne une augmentation de la part des ménages dont la personne de référence est chômeur (+3 points), salarié en contrat précaire - contrat à durée déterminée ou intérim - (+2 points) ou salarié en contrat à temps partiel (+2 points). Or ces ménages ont un niveau de non-motorisation plus de trois fois supérieur à celui des ménages dont les actifs sont employés en contrat à durée indéterminée ou qui exercent une activité non-salariée. D’autre part, on observe une forte augmentation de la part de ménages d'une personne (+4 points) et des familles mono-parentales (+2 points) qui explique cette résistance. Ces deux types de ménages sont, en effet, non-motorisés dans $21 \%$ des cas alors que ce chiffre n'est que de $4 \%$ pour les ménages comportant un couple. 
Parmi les ménages de retraités la non-motorisation a fortement reculé passant de $36 \%$ en 1990, à $26 \%$ en 1999. Les effectifs de ménages de retraités non-motorisés ont ainsi reculé de - $10 \%$ dans une période où le nombre total de retraités a augmenté de $+30 \%$. Le recul de la non-motorisation s'observe pour toutes les catégories de ménages, quels que soient leur taille, l'âge ou le sexe de la personne de référence. Il s'explique par l'impact conjoint de l'effet générationnel et de l'augmentation de l'espérance de vie en bonne santé. L’effet générationnel traduit l'arrivée progressive de l'automobile dans le mode de vie des ménages. Ainsi, les générations qui ont été peu motorisées au début de leur vie d'adulte se motorisent peu par la suite parce qu'elles « avaient déjà pris l'habitude de vivre sans » (Bodier, 1996). Par contre les générations arrivées à l'âge adulte à partir des années 1960 ont été motorisées dès cette période et arrivent aujourd'hui à la retraite avec des niveaux de motorisation nettement plus élevés que leurs aînés (Gallez 1995). L'augmentation de l'espérance de vie en bonne santé est une tendance continue en France au moins depuis le XIX ${ }^{e}$ siècle et permet aujourd'hui aux ménages âgés de conserver plus longtemps un accès à l'automobile. Ces deux tendances contribuent à un recul de la non-motorisation parmi les retraités et cet effet devrait se poursuivre durant la prochaine décennie.

Si la baisse de la part des ménages non-motorisés est inégale entre ménages d'actifs et ménages de retraités, elle l'est également dans l'espace. En effet, le recul de la non-motorisation a été plus fort dans les communes peu ou pas équipées de services. La part des ménages non-motorisés est ainsi passée de $10 \%$ à 6,5\% là où il n'y a aucun service à proximité. Dans les autres communes la baisse de la part des ménages non-motorisés n'est que de 2 points. Cette tendance au repli des ménages non-motorisés vers les espaces les mieux équipées en services s'observe aussi bien pour les ménages d'actifs que pour les ménages de retraités. Malgré cette tendance à la concentration, les ménages non-motorisés dans les espaces les mieux équipés, les retraités conservent une diffusion territoriale plus forte. En 1999, 9 \% des retraités non-motorisés résidant dans les communes qui ne disposent d'aucun service et $17 \%$ dans celles qui disposent seulement d'un service de proximité, contre respectivement $2 \%$ et $11 \%$ des actifs non-motorisés.

\section{LES ACTIFS NON-MOTORISÉS « FUIENT »}

\section{LES TERRITOIRES LES PLUS EXPOSÉS À LA DÉPENDANCE AUTOMOBILE, PAS LES RETRAITÉS}

L'inégale concentration dans les territoires de grande couronne des ménages d'actifs et de retraités non-motorisés traduit probablement un rapport différent de ces deux catégories de ménages à la dépendance automobile. En effet, nos analyses semblent montrer que les ménages d'actifs non-motorisés tendent à «fuir » les territoires les plus dépendants de l'automobile alors que ce phénomène ne touche pas les ménages de retraités (tableau 1).

On peut lire le tableau 1 de la façon suivante: Dans les communes qui ne disposent d'aucun service ( $1^{\text {re }}$ ligne), les ménages non-motorisés ne représentent que 2,2 \% de l'ensemble des ménages d'actifs en 1999, mais ils représentent 10,1\% des ménages sortants de ce type de commune et 2,2 \% des ménages qui y sont arrivés entre 1990 et 1999. Un même ménage figure à la fois parmi les sortants et les entrants s'il a effectué une migration résidentielle en provenance et à destination d'une commune de grande couronne entre 1990 et 1999.

De fait, l'analyse de la mobilité résidentielle nous montre une surreprésentation des ménages non-motorisés parmi les ménages d'actifs qui ont quitté leur commune de résidence entre 1990 et $1999^{4}$. Cette surreprésentation est particulièrement forte dans les communes peu ou pas équipées de services et de plus en plus faible à mesure que le niveau de service croît. Pour les communes non-équipées de services, la surreprésentation des non-motorisés parmi les sortants est la plus importante puisque les non-motorisés représentent $10,1 \%$ des sortants, mais seulement 2,2 \% de l'ensemble des ménages d'actifs. Pour les communes équipées de services de proximité,

4. Les sorties correspondent à l'ensemble des ménages en 1999 qui déclarent avoir changé de commune de résidence entre 1990 et 1999 et qui ont eu leur résidence précédente dans une commune de grande couronne. 
les ménages non-motorisés représentent 11,6\% des sortants contre 5,4\% des actifs résidant dans ces communes en 1999. Pour les deux autres niveaux de services cette différence atteint 3 points dans les communes équipées d’une grande surface et 2 points dans les communes équipées de bouquet de services.

\begin{tabular}{|c|c|c|c|c|}
\hline $\begin{array}{c}\text { Type de mobilité résidentielle } \\
\text { / Type de commune en grande }_{\text {couronne }}\end{array}$ & Ensemble & $\begin{array}{c}\text { Pas de mobilité }^{1} \\
\text { résidentielle }^{2}\end{array}$ & Sortants $^{3}$ & Entrants $^{4}$ \\
\hline $\begin{array}{c}\text { Aucun } \\
\text { équipement }\end{array}$ & 2,2 & 1,9 & 10,1 & 2,2 \\
\hline $\begin{array}{c}\text { Services } \\
\text { de proximité }\end{array}$ & 5,4 & 4,6 & 11,6 & 6,0 \\
\hline $\begin{array}{c}\text { Grande } \\
\text { surface }\end{array}$ & 8,0 & 6,5 & 13,0 & 9,8 \\
\hline $\begin{array}{c}\text { Bouquet } \\
\text { de services }\end{array}$ & 13,1 & 11,3 & 14,0 & 15,9 \\
\hline Ensemble & 10,0 & 8,3 & 13,2 & 11,7 \\
\hline
\end{tabular}

Tableau 1 : Part des non-motorisés parmi les ménages dont la personne de référence est un actif (en \%) selon leur mobilité résidentielle entre 1990 et 1999 (Source : Inventaire Communal 1998, Recensement 1999; calculs de l'auteur)

1 Ensemble des ménages résidants dans une commune de grande couronne en 1999.

2 Ménages résidants dans une commune de grande couronne en 1990 et n’en ayant pas changé en 1999.

3 Ménages résidants dans une commune de grande couronne en 1990 et en ayant changé pour une commune située ou non en grande couronne en 1999.

4 Ménages résidants dans une commune située ou non en grande couronne en 1990 et en ayant changé pour une commune située en grande couronne en 1999.

and 1999

Percentage of not-motorized workers households according to residential mobility between 1990

Les ménages d'actifs non-motorisés ont ainsi une tendance plus forte à changer de commune de résidence que les autres actifs. On observe que ce mouvement est différent selon les territoires : là où l'équipement en services est le plus faible et la dépendance automobile la plus forte l'ampleur des départs suggère un phénomène de fuite. Ceci renforce notre hypothèse du caractère répulsif des territoires les plus dépendants de l'automobile pour les ménages non-motorisés. Mais, l'absence de données longitudinales ne permet pas toutefois d'en apporter une démonstration plus complète.

L'observation de la mobilité résidentielle des ménages non-motorisés conduit à un second résultat : l'existence, pour les ménages pauvres de grande couronne, d'alternatives résidentielles plus centrales et dans des localisations résidentielles moins dépendantes de l'automobile. En effet, près de $51 \%$ des ménages non-motorisés qui ont quitté la grande couronne, et notamment les communes les plus dépendantes de l'automobile, sont allés s'installer à Paris ou en petite couronne. Ce constat va à l'encontre, au moins partiellement, de l'idée d'un périurbain de relégation peuplé de ménages pauvres non-motorisés contraints de rester dans des périphéries mal équipées et mal desservies et dans impossibilité de trouver dans les centres d'agglomération des logements à leur portée financière.

À l’inverse des actifs, les ménages de retraités non-motorisés, quel que soit le territoire considéré, ne sont pas surreprésentés parmi les ménages de retraités ayant quitté leur commune de résidence en grande couronne entre 1990 et 1999. Ces ménages semblent même fortement ancrés dans leurs localisations résidentielles puisque seulement $15 \%$ d'entre eux ont quitté leur commune de résidence en grande couronne entre 1990 et 1999. Ce chiffre est très en deçà de la mobilité résidentielle des retraités motorisés $(25 \%)$ et surtout de celle des actifs en général (44\%). Le très faible taux de déménagement des ménages de retraités non-motorisés pourrait témoigner de leur 
faible capacité à changer de localisations résidentielles à l'inverse de ce que l'on observe pour les actifs non-motorisés. Ce phénomène mériterait d'être étudié plus précisément, même s’il concerne une population peu nombreuse et qui ne devrait pas augmenter fortement, compte tenu de l'augmentation probable de la motorisation des retraités dans les générations futures.

\section{Les disparités de mobilité entre les ménages même motorisés sont particulièrement fortes dans les territoires les plus dépendants de l'automobile}

La motorisation ne constitue pas, pour les ménages résidant en grande couronne, une assurance face à la dépendance automobile. Ainsi, on constate pour certains ménages ne disposant que d'un seul véhicule, comme pour les ménages non-motorisés, un recours aux proches pour assurer certains de leurs déplacements ou un recours prononcé aux transports en commun pour se rendre au travail. Pour autant, ces dépendances restent très limitées pour les ménages motorisés. Dans $10 \%$ seulement des ménages mono-motorisés l'un des actifs se fait accompagner régulièrement, sur son lieu de travail, par un proche extérieur au ménage et $30 \%$ de ces ménages ont recours de façon régulière aux transports en commun toujours pour les navettes domicile-travail. Ces pratiques sont notamment le révélateur des faibles capacités financières de certains ménages motorisés.

Mais si ces pratiques restent marginales pour les ménages motorisés et au-delà de ces observations, d'autres analyses sur le niveau de motorisation et de mobilité automobile nous révèlent des faiblesses dont l'ampleur est significative montrant que même motorisés, les ménages modestes résidant en grande couronne francilienne sont fragilisés dans leur mobilité.

\section{LES MÉNAGES MOTORISÉS FACE À LA DÉPENDANCE AUTOMOBILE}

On observe tout d'abord que le niveau de motorisation des ménages résidant dans les communes les mieux équipées en services (disposant d'au moins une grande surface et/ou d'un bouquet de services) est en moyenne inférieur de 2 points à la moyenne régionale. À l'inverse, dans les communes peu ou pas équipées en services (aucun service ou seulement un service de proximité), le niveau de motorisation des ménages, à structure et caractéristiques similaires ${ }^{5}$, est jusqu’à 10 points supérieur à la moyenne de l'ensemble de la grande couronne. Le nombre de kilomètres parcourus en voiture particulière varie également selon le niveau d'équipement de la commune de résidence. Ainsi, un ménage habitant dans une commune ne possédant pas de services de proximité, parcourt en moyenne plus de $40 \%$ de kilomètres supplémentaires par rapport au niveau moyen enregistré en grande couronne pour le même type de ménage. À l'inverse un ménage résidant dans une commune équipée d'un bouquet de services parcourt en moyenne une distance près de - $6 \%$ inférieure à celle enregistrée en grande couronne pour le même type de ménage. Ces résultats témoignent bien du fait que les ménages de grande couronne sont confrontés à des situations très différentes en fonction des territoires où ils résident. Les exigences en matière d'équipement et de mobilité automobiles que traduisent ces moyennes augmentent quand le niveau de services des communes diminue.

5. Méthodologie : dans cette partie, pour mettre en évidence les différences de comportement de motorisation et de mobilité automobile entre les territoires et entre les ménages selon leur PCS, nous avons comparé pour chaque territoire et pour chaque PCS le niveau de ces deux indicateurs par rapport au niveau moyen constaté pour l'ensemble des ménages et pour l'ensemble de la grande couronne. Afin de neutraliser les effets d'autres caractéristiques fortement discriminantes à l'égard de ces deux indicateurs, nous avons opéré ces mesures au sein de catégories « homogènes » de ménages. Ainsi, les ménages ont été ventilés entre une centaine de catégories différentes, déterminées à partir de sept variables : sexe de la personne de référence, nombre de personnes du ménage, nombre d'actifs du ménage, pour les actifs nous avons également considéré la distance domicile travail, le fait d'être ou de ne pas être au chômage et la nature du contrat de travail (CDI, CDD, intérim, profession libérale etc.). De cette manière, nous avons par exemple une catégorie de ménages « qui compte trois personnes dont un actif, dont la personne de référence est un homme qui travaille en CDI à moins de $10 \mathrm{~km}$ de son travail ». 
Cette réponse graduée des ménages en fonction des services offerts par leur commune de résidence s'observe quelque soit leur PCS (tableau 2). De sorte que, les niveaux de motorisation et de mobilité automobile d'un ménage de cadre ou d'ouvrier, toutes choses égales par ailleurs, sont plus importants dans une commune qui ne dispose d'aucun service que dans une commune qui possède d'un bouquet de services. Pour autant, ce qui diffère pour chacune des PCS par rapport au modèle général, ce sont les niveaux moyens d'équipement et de la mobilité automobiles, mais également l'ampleur de leurs variations entre les territoires.

Ainsi, en ce qui concerne l'équipement automobile, les niveaux de motorisation des cadres et des professions intermédiaires sont toujours les plus élevés quels que soient les territoires considérés. Les ménages de retraités se placent dans une situation intermédiaire entre les ménages d'actifs les plus aisés (cadres et professions intermédiaires) et les ménages les plus modestes (employés et ouvriers). Dans les communes mieux équipées, les ménages d'ouvriers ou d'employés profitent des ressources locales plus abondantes et adoptent un niveau d'équipement automobile particulièrement réduit, très en dessous de la moyenne régionale. Dans, les communes qui disposent de peu de ressources locales les ménages modestes sont contraints d'investir beaucoup plus fortement dans leur équipement automobile et adoptent des niveaux de motorisation très supérieurs à la moyenne régionale, et tendent à se rapprocher de ceux des cadres. De leur côté, ces derniers adoptent quelque soit la commune un niveau de motorisation élevé, qui hausse encore sensiblement lorsque le niveau de ressource locale est plus faible.

\begin{tabular}{|c|c|c|c|c|c|c|}
\hline $\begin{array}{c}\text { PCS / } \\
\text { Type de commune }\end{array}$ & Cadres & $\begin{array}{c}\text { Professions } \\
\text { intermédiaires }\end{array}$ & Employés & Ouvriers & Retraités & Ensemble \\
\hline Aucun équipement & 17 & 14 & 9 & 8 & 9 & 9 \\
\hline $\begin{array}{c}\text { Services } \\
\text { de proximité }\end{array}$ & 13 & 6 & 0 & 1 & 4 & 8 \\
\hline $\begin{array}{c}\text { Grande surface } \\
\begin{array}{c}\text { Bouquet } \\
\text { de services }\end{array}\end{array}$ & 6 & -1 & -7 & -8 & -2 & -2 \\
\hline
\end{tabular}

Tableau 2 : Différences (en \%) par rapport à la moyenne des niveaux de motorisation des ménages selon leur PCS et leur localisation résidentielle (Source : DREIF 1999, Inventaire Communal 1998, Recensement 1999; calculs de l'auteur) residential localization

Pour ce qui est de la mobilité automobile quotidienne, le constat est assez dissemblable de celui que nous venons de dresser concernant l'équipement automobile (tableau 3). Les niveaux de mobilité des ménages aisés sont certes supérieurs à ceux des ménages modestes, pour autant ce sont les premiers qui affichent la plus forte sensibilité aux caractéristiques des territoires. Ainsi, les cadres qui parcourent un nombre de kilomètre relativement faible dans les communes équipées de bouquet de services augmentent très fortement cette mobilité quand ils résident dans une commune plus faiblement équipée en services. À l'inverse, les ménages modestes ont un niveau de mobilité automobile qui augmente faiblement avec de faibles ressources locales, comme si en raison de leurs ressources et l'effort déjà consentit pour élever leur niveau d'équipement automobile ils ne pouvaient concéder que parcimonieusement une augmentation des kilomètres parcourus en voiture.

Ainsi, nous avons mis en évidence par l'analyse de ces indicateurs de motorisation et de mobilité automobile deux types de comportement. Celui des ménages les plus aisés qui, face à des ressources locales réduites, haussent modérément leur niveau d'équipement automobile déjà relativement élevé mais surtout ont un niveau de mobilité automobile qui explose. Ce modèle est également à des degrés différents celui des ménages de retraités. Par rapport aux ménages les plus aisés, les 
ménages modestes, face à des ressources locales réduites, sont contraints de hausser un niveau d'équipement automobile relativement bas mais ne haussent que très faiblement leur mobilité automobile. La fragilité des ménages modestes motorisés face à la dépendance automobile dans les espaces périurbains semble se traduire plus nettement par leur niveau de mobilité automobile plutôt que par leur niveau d'équipement.

\begin{tabular}{|c|c|c|c|c|c|c|}
\hline $\begin{array}{c}\text { PCS / } \\
\text { Type de commune }\end{array}$ & Cadres & $\begin{array}{c}\text { Professions } \\
\text { intermédiaires }\end{array}$ & Employés & Ouvriers & Retraités & Ensemble \\
\hline Aucun équipement & 53 & 72 & 8 & - & 39 & 44 \\
\hline $\begin{array}{c}\text { Services } \\
\text { de proximité }\end{array}$ & 33 & 17 & 4 & 19 & 21 & 39 \\
\hline Grande surface & 8 & -3 & -30 & -4 & 5 & 8 \\
\hline $\begin{array}{c}\text { Bouquet } \\
\text { de services }\end{array}$ & -9 & -7 & -27 & -21 & -15 & -7 \\
\hline
\end{tabular}

Tableau 3 : Différences (en \%) par rapport à la moyenne régionale du nombre de kilomètres parcourus quotidiennement en voiture par les ménages selon leur PCS et leur localisation résidentielle (Source : DREIF 1999, EGT 2001, Inventaire Communal 1998 ; calculs de l'auteur)

Percentage differences to average of households automobile mobility levels according to occupation and residential localization

\section{Trois scénarios et leurs impacts sur la place de l'automobile dans les espaces périurbains}

Face à cette situation, où un certain nombre de ménages non-motorisés sont marginalisés face à la dépendance automobile en raison de la faiblesse de leurs capacités financières ou de leurs capacités fonctionnelles, nous avons mesuré l'impact potentiel des évolutions de la diffusion des services aux ménages en grande couronne. L'utilisation de telles simulations s’inspire directement du travail d'E. Korsu et M.-H. Massot (2006) qui ont utilisé cette méthodologie en Île-de-France pour évaluer l'impact d'un rapprochement des lieux de résidences par rapport aux lieux d'emploi sur l'usage de l'automobile. Nous avons ainsi simulé trois scénarios dont le premier est une évolution au fil de l'eau et les deux autres en rupture par rapport à la situation actuelle vont dans le sens d'un allégement du niveau de la pression automobile. La première de ces ruptures est une offre de services de proximité dans toutes les communes. La seconde aurait pour objectif de limiter ou contrôler l'étalement des populations dans les espaces périurbains et de favoriser l'accès aux services en transport en commun en relocalisant ceux qui ne le sont pas à moins de 5 ou 10 minutes en transport en commun d'un bouquet de services.

Pour chacun de ces scénarios, nous évaluerons leur impact probable sur la part du volume global du parc automobile et du nombre de kilomètres parcourus en grande couronne. L'utilisation de ces deux variables comme indicateur de l'intensité de l'allégement du poids de la dépendance automobile sur les ménages s'est faite par défaut. En effet, l'échantillon de l'EGT ne nous permettait pas de faire un décompte précis par type de territoire du nombre de ménages en dessous du " seuil de marginalité » qui bénéficierait directement d'un allégement du poids de leur dépendance à l'automobile en vertu de ces scénarios. Nous avons donc réalisé une estimation un peu plus large mais qui permet de d'évaluer l'efficacité de ces scénarios. 


\section{ÉVOLUTION AU « FIL DE L'EAU ${ }^{6} »$}

Dans ce premier scénario, nous avons simulé l'impact d'une évolution de la diffusion territoriale des niveaux de services dans les communes de grande couronne pour la prochaine décennie équivalente à celle observée au cours de la décennie passée ${ }^{7}$. On constate que cela permettrait, toutes choses égales par ailleurs, un allégement de la pression automobile. En effet, dans ce scénario le nombre de communes équipées d'une grande surface se renforce fortement et malgré un recul des services de proximité, un plus grand nombre de personnes devrait bénéficier d’un niveau supérieur de ressources locales. De cette manière et toutes choses égales par ailleurs, le parc automobile diminuerait d'environ 46000 unités, soit 2,4\% du parc et le nombre de kilomètres parcourus baisserait de 900000 kilomètres soit 1 \% des distances totales parcourues en grande couronne.

\section{UNE OFFRE DE PROXIMITÉ POUR TOUS ${ }^{8}$}

Si l'on alloue à l'ensemble des ménages des services de proximité dans leur commune de résidence, on peut évaluer, toutes choses égales par ailleurs, à -5800 de voitures, la baisse du parc automobile soit une diminution de $-0,3 \%$ du parc total. En ce qui concerne les kilomètres, la baisse serait de $-3,3 \%$ soit -1,8 millions de kilomètres par jour. Par ailleurs, cela permettrait à près de 9000 ménages non-motorisés de bénéficier dans leur commune de résidence de services de proximité ce qui, nous l'avons vu, constitue pour ces ménages une amélioration significative de leurs approvisionnements.

\section{TOUS LES MÉNAGES RÉSIDENT À PROXIMITÉ D'UN BOUQUET DE SERVICE ACCESSIBLE EN TRANSPORT EN COMMUN EN MOINS DE 5 OU 10 MINUTES ${ }^{9}$}

Dans cette situation, le niveau de motorisation de la grande couronne baisserait de $-1,2 \%$ soit environ -23 700 voitures. En termes de distances parcourues cela aurait un impact considérable puisque cela tendrait à réduire de $-7,6 \%$ le nombre moyen de kilomètres parcourus par ménage, soit -4 millions de kilomètres au total. Si l'on raisonne avec un périmètre un peu plus large que celui que nous venons d'étudier et que nous localisons les populations à moins de 10 minutes en moyenne des services, la motorisation recule de $-0,6 \%$ et le nombre de kilomètres de $-4,3 \%$.

6. Méthodologie : Pour cette simulation nous avons calculé, pour 1999, le niveau moyen de motorisation et le nombre de kilomètres parcourus pour chacune des catégories de ménages que nous avons définies précédemment dans chacun des niveaux de services (commune sans services, avec des services de proximité, avec une grande surface, avec un bouquet de services). Nous avons ensuite remplacé, pour les ménages résidant dans une cinquantaine de communes équipées de services de proximité leurs niveaux de motorisation et le nombre de kilomètre qu'ils parcourent quotidiennement en voiture particulière par la valeur moyenne de ces deux indicateurs dans une commune qui ne dispose d'aucun service. Nous avons également remplacé les valeurs de motorisation et de mobilité automobile pour les ménages résidant dans une centaine de communes équipées de services de proximité par la valeur moyenne de ces deux indicateurs dans une commune équipée d'une grande surface qui ne dispose d'aucun service. Ces réaffectations devraient refléter l'évolution des services aux ménages dans la décennie à venir, si l'on en croit les évolutions observées ces 20 dernières années.

7. Recul de $10 \%$ de la diffusion territoriale des services de proximité, progression de $25 \%$ des grandes surfaces et stagnation de la diffusion territoriale des bouquets de services (Motte, 2006).

8. Méthodologie : Pour cette simulation nous avons calculé, pour 1999, le niveau moyen de motorisation et le nombre de kilomètres parcourus pour chacune des catégories de ménages que nous avons définies précédemment dans chacun des niveaux de services (commune sans services, avec des services de proximité, avec une grande surface, avec un bouquet de services). Nous avons ensuite remplacé, pour chaque ménage dont la commune de résidence ne dispose d'aucun service, son niveau de motorisation et le nombre de kilomètre qu'il parcourt quotidiennement en voiture particulière par la valeur moyenne de ces deux indicateurs dans une commune dont le niveau de service est un service de proximité.

9. Méthodologie: Pour cette simulation nous avons calculé, pour 1999, le niveau moyen de motorisation et le nombre de kilomètres parcourus pour chacune des catégories de ménages que nous avons définies précédemment dans un périmètre de 5 et 10 minutes en transports en commun autour d'un bouquet de services.

Nous avons ensuite remplacé, pour chaque ménage résidant à plus de 5 ou plus de 10 minutes de son univers de services, son niveau de motorisation et le nombre de kilomètre qu'il parcourt quotidiennement en voiture particulière par la valeur moyenne de ces deux indicateurs dans une commune située dans un périmètre de 5 et 10 minutes en transports en commun autour d'un bouquet de services. 
En termes de bilan pour ces scénarios, on peut noter qu'une mesure telle qu'une généralisation des services de proximité à l'ensemble des communes de grande couronne aurait un impact à peine plus important que l'évolution au fil de l'eau, ce qui montre la force des dynamiques internes et le peu d'effet d'une telle mesure. D'autant plus, que l'on peut douter de la portée d'une telle mesure pour répondre aux attentes des populations locales (Héry, 2006). Une limitation de l'étalement dans un périmètre de 10 minutes en transports en commun autour des principales offres de services aurait un effet un peu plus important mais révèle que contrecarrer, ou encadrer les dynamiques actuelles est particulièrement difficile. D'autre part, le premier scénario nécessiterait un financement significatif et continu pour une efficacité restreinte, tandis que notre seconde rupture nécessiterait une volonté politique également forte et continue ce qui parait aujourd'hui difficile. Enfin, l'impact de ces différents scénarios est assez peu différencié entre les PCS ou entre les actifs et les retraités. Aucune des simulations que nous avons faites ne nous permet d'affirmer qu'une évolution ou une mesure politique agissant sur la diffusion des services ou sur la localisation des populations par rapport aux services favoriserait ou handicaperait significativement l'une ou l'autre des catégories de ménages quant à leur équipement automobile ou leur mobilité.

\section{Conclusion}

Le rôle structurant de l'automobile dans les espaces périurbains tend à induire de fortes disparités entre les ménages. En effet, l'automobile et plus largement la mobilité automobile n'est pas à la portée de toutes les bourses et nécessite des capacités fonctionnelles qui s'amenuisent avec l'âge. Or si l'on fait l'hypothèse d'une relégation dans les espaces périurbains des ménages les plus modestes rejetés dans les marges par le coût du logement très supérieur dans les centres et la difficulté de ce fait pour les ménages qui y sont installés, notamment les plus âgés, d'envisager une migration résidentielle en direction des centres, alors les disparités de mobilité entre les ménages périurbains devraient s'accroître fortement.

Nos résultats démentent en un sens cette hypothèse pour la grande couronne francilienne. En effet, les populations non-motorisées dont la mobilité est fortement marquée par leur absence d'équipement automobile voient leur nombre diminuer sensiblement partout dans les espaces périurbains. Le vieillissement de la population ou le maintien du chômage à un niveau élevé n'ont pour le moment qu'un effet marginal, alors qu'on observe des trajectoires résidentielles en direction des centres pour de nombreux ménages non-motorisés qui « fuient », dans une certaine mesure, les territoires périurbains les plus dépendants de l'automobile. Pour autant, l'exclusion d'un nombre plus important de ménages par de fortes disparités de mobilité est plus certainement à aller chercher du côté des ménages motorisés. Les plus modestes d'entre eux peinent à tirer pleinement profit de leur équipement automobile, notamment dans les territoires où ils en ont le plus besoin. Dans ces territoires dotés de plus faibles ressources en termes de commerces et services, ces ménages ont des niveaux de mobilité très inférieurs aux autres ménages et partagent avec les ménages non-motorisés certaines de leurs difficultés à faire face aux fortes «injonctions à la mobilité automobile» (Wenglenski, 2003). Dans les territoires mieux dotés en ressources, ces difficultés ne disparaissent pas mais ne se posent pas avec la même acuité.

Les territoires périurbains tout comme les populations qui les composent ne sont pas homogènes et il paraît nécessaire au vu de notre étude de les envisager en prenant en compte ces deux dimensions. Différents scénarios simulés montrent ainsi qu'une politique globale qui concernerait indistinctement territoires et populations n'aurait qu'un impact négligeable ou nécessiterait des moyens trop importants. On voit bien que l'efficacité ne peut ici passer par des mesures identiques entre les ménages non-motorisés et les autres, tandis que les interventions sur les territoires sont nécessairement différentes entre communes plus faiblement peuplées, ne disposant pas de ressources locales et celles plus densément peuplées disposant de ressources locales riches. 


\section{Bibliographie}

Berger (M.), 2004. - Les périurbains de Paris. De la ville dense à la métropole éclatée?, Paris, CNRS, coll. «Espaces \& Milieux », 314 p.

Bodier (M.), 1996. - «La voiture : une habitude qui se prend jeune », INSEE Première, 474, 4 p.

CARRE (S.), 2005. - La construction newve en Île-de-France de 1990 à 2004, Paris, Direction Régionale de l'Équipement Île-de-France, 15 p.

Coutard (O.), Dupuy (G.), Fol (S.), 2002. - « La pauvreté périurbaine : dépendance locale ou dépendance automobile? ", Espaces et Sociétés, p. 155-175.

Dupuy (G.), 1999. - La dépendance automobile. Symptômes, analyses, diagnostic, traitements, Paris, Anthropos, $160 \mathrm{p}$

GALLEZ (C.), 1995. - «Une nouvelle perspective pour la projection à long terme des comportements d'équipement et de motorisation ", Recherche, Transports, Sécurité, 48, p. 3-14.

Guilluy (C.), Noyé (C.), 2004. - Atlas des nouvelles fractures sociales en France, Paris, Autrement.

Godard (F.), 2001. - La ville en mouvement, Paris, Gallimard, coll. « Découvertes », 127 p.

HÉRY (D.), 2006. - Dynamiques de périurbanisation et systèmes de mobilités des ménages, le cas du grand périurbain clermontois, Créteil, Mémoire de Master, Université de Paris 12 - Val de Marne, 59 p.

Hivert (L.), PÉAn de Ponfilly (J.), 2000. - Inégalités d'accès à l'automobile - Disparités d'équipement et d'usage entre les ménages les plus pauvres et les plus riches, Arcueil, Rapport de convention, INRETS, $80 \mathrm{p}$.

ILLICH (I.), 1973. - Énergie et équité, Paris, Seuil, 33 p.

INSEE, 2001. - «Inventaire Communal 1998 », Synthèses, 52, 167 p.

INSEE, 1996. - «Une nouvelle approche territoriale de la ville», Économie et Statistique, p. 294-295.

JAILLET (M.-C.), 1985. - «Quelques paradoxes périurbains », dans Les périphéries urbaines, Actes du colloque d'Angers des 6 et 7 décembre 1984, 270 p.

JALABERT (J.), 1985. - «Espaces et populations dans les périphéries urbaines », dans Les périphéries urbaines, Actes du colloque d'Angers des 6 et 7 décembre 1984, 270 p.

Kaufmann (V.), Guidez (J.-M.), 1998. - Les citadins face à l'automobilité. Les déterminants du choix modal, Lyon, CERTU-ADEME-UTP, $121 \mathrm{p}$.

Konsu (E.), Massot (M.-H.), 2006. - « Rapprocher les ménages de leurs lieux de travail : les enjeux pour la régulation de l'usage de la voiture en Île-de-France ", Les cahiers scientifiques du transport, 50, p. 61-90.

Massot (M.-H.), Roy (E.), 2004. - Lieu de vie - Lieu de travail. 25 ans d'évolution de la distance au travail, Arcueil, Rapport, INRETS, 144 p.

Mотте (B.), 2006. - La dépendance automobile pour l'accès aux services aux ménages en grande couronne francilienne, Thèse de doctorat en géographie, Université de Paris 1 - Panthéon Sorbonne, 380 p.

Orfeuil (J.-P.), 2004. - «Accessibilité, mobilité, inégalités : regards sur la question en France aujourd'hui », dans Orfeuil (J.-P.), Transports, pawvretés exclusions. Pouvoir bouger pour s'en sortir, La Tour-d'Aigues, Éd. de l'Aube, p. 27-47.

Rougé (L.), 2005. - Accession à la propriété et modes de vie en maison individuelle des familles modestes installées en périurbain lointain toulousain, les «captifs » du périurbain, Toulouse, Thèse de doctorat en géographie, Université de Toulouse 2 - Le Mirail, 381 p.

Wenglenski (S.), 2003. - Une mesure des disparités sociales d'accessibilité au marché de l'emploi en Île-deFrance, Créteil, Thèse de doctorat en urbanisme, aménagement et politiques urbaines, Université de Paris 12 - Val de Marne, 373 p.

Cet article a été reçu le le 18 juin 2007 et définitivement accepté le 28 novembre 2007. 